\title{
Mathematical Basis for Modeling Swimmer Power Output in the Front Crawl Tethered Swimming: An Application to Aerobic Evaluation
}

\author{
D.M. Pessôa Filho ${ }^{1,2}$ and B.S. Denadai* ${ }^{2}$ \\ ${ }^{I}$ Department of Physical Education, College of Science, UNESP Bauru, São Paulo, Brazil and ${ }^{2}$ Human Performance \\ Laboratory, UNESP Rio Claro, São Paulo, Brazil
}

\begin{abstract}
The purposes of this manuscript were to discuss the propelling hydrodynamics in human tethered swimming, and its application to strength evaluation and to approach power-time limit model $\left(\mathrm{P}-\mathrm{t}_{\mathrm{Lim}}\right)$ in tethered-crawl $\left(\mathrm{Pu}\right.$ Teth $\left.\mathrm{t}_{\mathrm{Lim}}\right)$. Physiological and mechanical characteristics of tethered and free swimming have shown to be very similar, and thus tethered swimming is a reliable condition for evaluation and training. Hydrodynamics assumptions for hydrofoil in free swimming were applied for tethered swimming to support the power-time limit model reproduction. Critical power in crawl-tethered $\left(\mathrm{Pu}_{\text {TethCrit }}\right)$ was shown to be closely related to the critical velocity determined during free swimming and performance of competitive distances ranged from 200 to $1500-\mathrm{m}$. These results recognize the $\mathrm{Pu}_{\mathrm{Teth}} \mathrm{t}_{\mathrm{Lim}}$ model as a suitable method to reproduce $\mathrm{P}-\mathrm{t}_{\mathrm{Lim}}$ model in swimming, but the reliability of the $\mathrm{Pu}_{\mathrm{TethCrit}}$ as an index of aerobic capacity, and training intensity must be explored in further researches.
\end{abstract}

Key Words: Active drag force, Tethered swimming, Hydrofoil mechanics, Power-time limit model.

\section{INTRODUCTION}

This study considers tethered swimming conditions as one of the most sport-specific ergometers for swimmers. Tethered swimming refers to a condition where the swimmer is connected to weights via rope-and-pulley system or to another machine where swimming body thrust is recorded by means of a strain gauge (load cell, force transductor linked to a dynamometer, and so on) $[1,2,3]$. To establish fully tethered swimming conditions, added weight must prevent swimmer to move forward $(\mathrm{v}=0)$ [1]. According to Martin et al. [4], this case may be written as:

$$
\mathrm{Ft}+\mathrm{Fr}-\mathrm{Fp}=0
$$

where $\mathrm{Ft}$ is the tether force, that is, the external load which is attached to swimmer and counteracts forward body displacement in water; Fr is the active drag force, which is originated by the swimmer body displacement on fluid surface, and opposite forward movement; and Fp is the hydrofoil propelling force, that is, the thrust created during the pushing back movement of the stroke to propel swimmer body forward, overcoming $\mathrm{Fr}$ and counteracting Ft. The maximum (tether) force recorded at zero velocity has been recognized as a measurement of the maximum propulsive force that, theoretically, corresponds to the (propelling) force necessary to overcome the resistance at maximum free-swim velocity [1]. The reasonable accuracy in approaching real propulsive situation has been subjected to criticisms, since the models are not refined enough to account either for adjustments of stroke kinematics similar to free swimming conditions, or for a careful explanation of how the force evaluated relates to performance, and how it would be managed by coaches to design specific strength training [2,

*Address correspondence to this author at the Human Performance Laboratory, UNESP Rio Claro, São Paulo, Brazil; E-mail: bdenadai@rc.unesp.br
$3,5,6]$. There is a tendency to point tether force as an exaggerated version of the propelling force swimmer is able to achieve, since backward velocity of the hydrofoil relative to the water decreases as forward body velocity increases [4], which causes a drop in propulsive force.

Thus, the purposes of this manuscript were (a) to discuss some general aspects of the propelling hydrodynamics model in human tethered swimming; (b) to analyze the application of the fundamental mechanics of human tethered swimming in strength evaluation and training and; (c) to approach power-time limit model in tethered-crawl conditions and the relationships of critical power (PC) with critical velocity $(\mathrm{CV})$ and performance in a range of free-swim competition races.

\section{THEORETICAL SUPPORT TO APPLY TETHERED SWIM AS A TOOL TO FORCE EVALUATION AND DAILY TRAINING STIMULUS}

Tethered swimming has been evoked as the most widely test used to measure pulling force performed in swimming $[7,8]$, since it may be considered a sport-specific device to simulate swimming characteristics with respect to environment [9], stroke mechanics [10], physiological aspects [11], and body anthropometric and morphological (size, shape, body weight and height) influence on propelling forces formation [1]. This could be concluded from the observations that fully tethered swimming at a pace between $85-100 \%$ of maximal stroke rate induces electromyographic (EMG) patterns for arm musculature similar to free swimming [12]. Even, Yater et al. [7] have shown the existence of a relationship between the mean maximal force of tethered swimming and the free style velocity; and Costill et al. [13] found a significant correlation $(\mathrm{r}=0.84)$ between swimming power measured via tether system in the water and 25-yard swimming. Kjendlie and Thorsvald [14] reported that tethered swimming is a reliable method to estimate the maximal teth- 
ered swimming force, since test-retest measurements produced high correlation coefficients and low coefficients of variations from trials performed in different days and periods of the day by high and middle-skill swimmers, under un- and familiarized conditions.

Regarded as a specific training device to force development, tethered swimming has been claimed a suitable tool, although the relationship between force increase and swimming performance improvement is the main argument to constrain its application, or another strength training method (i.e., land strength training), on regular training planning. Another factor is relative to load reference and adjustments in tethered swimming to match the specificity of propelling force requirements in different strokes and competition races during free style swimming. However, tethered swimming may be considered a force controlling environment, which ensures a better distribution of hand force and velocity during the stroke cycle. This force controlled condition, in the $[15,16]$ or out of pool [17], would improve the dynamic characteristics of stroke, resulting in the ability to convert muscle power potential into stroke specific power. A decrease in the stroke length with no change or a slight increase in stroke rate, and the trend to a straightlined backward motion of the hand are observed in the swimming tethered condition as compared to the actual stroke [18]. Interestingly, those changes are also verified with increasing free swimming velocity [19], but technical adaptations of the stroke seem to be different in the tethered and non-tethered (free swimming) conditions to match the requirements of force and keep performance unchanged [7, 19]. In the tethered condition, a greater rate of force development is able to be achieved on a given time during propulsive phases [8], whereas in the free swimming the superposition of both arms is the factor related to a better power output and is associated to a longer duration of the push phase [20]. These differences have been shown to impair an effective transfer of the strength gained in tethered training to enhance propelling force during free swimming [18].

Pessôa Filho and Monteiro [21] aimed to apply the active drag at maximal swim velocity as load parameter for weight training to improve swimming performance. They have trained twelve swimmers that were undergone to training ten weeks in tethered and non-tethered swimming conditions. For tethered condition they used the following protocol: 10 sets, 30s of rest with respective stroke rate and load that of drag measure condition (50 meters distance at maximal swim crawl velocity [22]). The training in non-tethered condition followed regular protocol in the pool. Trained tethered swimming group showed improvements in front-crawl swimming velocity (1.37\%), and stroke rate (4.12\%). Even, this group showed decreases in the active drag force $(-6.76 \%)$, stroke distance $(-2.33 \%)$, and drag coefficient $(-9.04 \%)$. Thus, they could conclude that these changes are specifics to improvements in swimming technique and performance, and that active drag is a suitable load parameter for weight training in swimming. These results are in agreement with those reported to Toussaint and Vervoon [23] from free-swimming strength training protocol in the MADsystem. They showed an improvement of $2.28 \%$ in free swimming maximal velocity on 50-m race for training group after 10 weeks of training. Based on results obtained, Pessôa Filho and Monteiro [21] attributed the rise in swimming ve- locity to a small increase in the stroke rate, that was accomplished with no, or a slight decrease in the stroke length, whereas Toussaint and Vervoorn [23] explained that the increasing in free swimming velocity was a result of the capacity to perform more work per stroke, since measured power did increase and stroke rate reduced. As the stroke rate could be a scaling factor of the amount of propulsive force [24], both studies attributed the training related changes to technical ability that, according to Wakayoshi et al. [25], is the increase stroke length at submaximal same swimming velocity due to increasing in propelling efficiency or a decrease in drag resistance.

\section{FUNDAMENTAL DYNAMIC RELATED TO TETH- ERED SWIMMING}

Mechanical assumption for hydrofoil states that push-off against an amount of water always result in a certain wasted fraction of the power output that is greater as smaller is the difference of pressure between the sides of hydrofoil which, in turn, is induced by its backward velocity [26]. Hence, in each stroke, the applied force $(F)$ causes a backward velocity change $(\Delta v)$ in a certain amount of water $\left(\mathrm{M}_{\mathrm{w}}\right)$. This power transferred to the masses of water $\left(\mathrm{P}_{\mathrm{k}}\right)$ is not useful for propulsion [26, 27] and equals:

$$
P_{k}=f \cdot 1 / 2 M_{w} \cdot \Delta v^{2}
$$

were $\mathrm{f}$ is the stroke frequency. Although, as hydrofoil (mainly hand) has a backward velocity (u) with respect to the water, the difference of water pressure formed between the front and back sides induces to a reaction force, often called propelling drag force (Frp) [27, 28, 29], corresponding to:

$$
F_{r p}=1 / 2 C x_{H y d r o f} \cdot \rho \cdot S_{H y d r o f} \cdot u^{2}
$$

where $\mathrm{Cx}_{\text {Hydrof }}$ is the drag coefficient, $\mathrm{p}$ is the water density, and $\mathrm{S}_{\mathrm{Hydrof}}$ the plane area of the hydrofoil.

For free swimming, at the beginning of the race, the propulsive force (Frp) must exceed the active drag force (Fr) as much as possible [4]. However, as swimmer's velocity increases, the drag force increases as well (a quadratic increase because it is related to the square of swimming velocity - as stated below in equation 4), and a steady state velocity is reached. Assuming a uniform change of momentum of a certain amount of water, leading to a backward displacement of some mass of water with a given velocity in each and every stroke and a constant forward velocity of swimmers, the opposing drag force equals the propulsive force [26]:

$$
F_{r p}=F r=\frac{1}{2} C x_{B o d y} \times \rho \times S_{B o d y} \times v^{2}
$$

where $\mathrm{Cx}_{\mathrm{Body}}$ is the drag coefficient for the entire body, $\mathrm{p}$ is the water density, $S_{\text {Body }}$ the cross-section area of the body exposed to the fluid, and $v^{2}$ is the quadratic of the swimmer velocity.

Total active drag force (Fr) in swimming is determined by the pressure and friction drag and the wave-making resistance. According to Miller [30] and Barthels [31], theoretical basis of this resistance are: (a) pressure drag that is determined mainly by the cross-sectional area of the swimmer. This results from the separation of the boundary layer of 
fluid adjacent to the body surface, which reverses its flow and breaks away, causing an eddying of irregular flow next to the body downstream sides. This creates a higher pressure zone at the leading surfaces than in the trailing ones, thus resulting in a suction effect which magnitude depends on the size, shape, velocity and position of the swimmer relative to the fluid surface; (b) the friction drag, which depends on the friction between skin and water and will theoretically increase as the total skin surface increases. It refers to a retarding force acting on the swimmer by the boundary layer of water flowing backwards along the surfaces of the body which move forward through the water; and (c) wavemaking system, that is the result of the pile up trends of the water in front of swimmer and the hollows formed behind him, which creates an additional resistance by deformation of the surface and formation of bow waves. The wave motion over the body changes the flow pattern around it, as the wall stress of the traveling wave increases with nonstreamlined motion on fluid. With that, the formation of turbulence in the boundary layer of body increases too, leading to a rise in the amount of drag force [32].

Relationship between forward (Frp) and backward (Fr) forces gives an approximation of the statements proposed by equation 2, hence:

$$
\begin{gathered}
\frac{1}{2} C x_{\text {Hidrof }} \times \rho \times S_{\text {Hidrof }} \times \mu^{2}=\frac{1}{2} C x_{\text {Body }} \times \rho \times S_{\text {Body }} \times v^{2} \\
\frac{\mu}{v}=\sqrt{\frac{C x_{\text {Body }} \times S_{\text {Body }}}{C x_{\text {Hidrof }} \times S_{\text {Hidrof }}}}
\end{gathered}
$$

Equation 5 shows a notion of how the mechanism of propulsion only uses a part of the total power output produced by the swimmer to overcome active drag (Fr), and that other part is dissipated giving acceleration to a mass of water $\left(\mathrm{P}_{\mathrm{K}}\right)$. That is, a rough approach of the propelling efficiency (ep), defined by the ratio of the useful power $(\mathrm{Pu})$ to the total power output (Po) [26, 27, 29]:

$$
e_{p}=\frac{P u}{P o}=\frac{F r \cdot v}{(F r \cdot v)+P_{K}}
$$

Ep has been suggested as an index of swimming technique ability that might be the result of a large forward resultant vector, which is highly effective to propulsion and associated to low energy cost [29].

To evaluate differences in force and stroke patterns, Toussaint [33] proposed that records of stroke characteristics [distance per stroke (ds) and stroke frequency (fs)] must be related to hydrodynamics stroke mechanics, in the free swimming condition, to give work per stroke (As) and work per distance (Ad) using the formulae:

$$
A s=\frac{P o}{f s}
$$

$$
A d=\frac{A s}{d s}
$$

Since Ad dimension should be noted in Newton, it can be rewritten as:

$$
F p_{\text {Total }}=\frac{F r p(N)}{\operatorname{ep}(\text { decimal })}
$$

Ad or $\mathrm{Fp}_{\text {Total }}$ is assumed to be the amount of force produced from contractile elements to the stroke task [33]. Analogue to equation 8 , the parameter "ds" can be substituted by stroke length relative to swimmer (Ls) in order to satisfy tethered swim conditions. Thus:

$$
A s=\left(\frac{F r p}{e p}\right) \times L s
$$

and:

$$
\left(\frac{F r p}{e p}\right) \times L s=\frac{P o}{f s} \rightarrow P o=\left(\frac{F r p}{e p}\right) \times(L s \times f s)
$$

If $\mathrm{Ls} \times$ fs equals to hydrofoil velocity, then equation 11 gives:

$$
P o=\left(\frac{F r p}{e p}\right) \times v_{\text {Hidrof }}
$$

This equation satisfy tethered swimming overall power output measurements from active muscle, and approach total power output produced during free swimming condition, as long as, Frp in tethered swim match the specificity of overcoming a resistance similar to active drag in free condition. According to Toussaint et al. [22], tethered swimming condition could be considered, from a free swimming perspective, an external load that increases force against swimmer body direction, promoting: (a) reduction of swimmer velocity, which could approaches zero as mechanical power used beneficially to overcome active drag is expended giving a kinetic energy change to masses of water, and so (b) if no propulsion was generated, propelling efficiency approaches zero too.

Therefore, to consider the specificity of equation 12 , we assume from deductive analysis of hydrofoil mechanism of force production:

a) hand velocity reduction relative to the swimmer body; If the hydrofoil velocity (u) with respect to swimmer body velocity ( $v$ ) is $u+v$, so with no body toward movement $u$ was reduced and this also results in a less amount of wasted power, because mass of water velocity variation would be either small [26];

b) a part of overall mechanical power is used beneficially to overcome load resistance;

c) another part gives a kinetics energy change to masses of water, as a result of pushed back movement;

d) intensity of external resistance must overload a known free swimming condition, and only the load respective to active drag in a given swim velocity should accomplish the force parallelism assumption at both conditions. 
Finally, the resistance specificity and the magnitude of propulsive force vector distribution in a range of pulling pattern would seem different in tethered swimming compared to free swimming. These differences are not markedly as pointed out by Schleihauf [34] and Olbrecht and Clarys [35] with respect to strength training on land, once line of motion in tethered condition simulates with reasonable approximation, most of the pattern motion $[36,37]$ and muscle activation [12] encountered in free swimming. Anyway, whereas a smoothly force application is a feature of stroke pattern in tethered swimming, because of external constant loading, that would differ from free swimming, the load respective to active drag force, or a fraction of it, will conjecture the nearest force management between conditions.

\section{APPLING POWER OUTPUT PRODUCED DURING TETHERED SWIMMING CONDITION FOR MODEL- ING POWER-TIME LIMIT RELATIONSHIP IN SWIMMING: A POSSIBILITY}

The application of power-time limit model $\left(\mathrm{P}-\mathrm{t}_{\mathrm{Lim}}\right)$ is an attempt to assess the physiological potential of the athlete in the specific sport environment. It is a field test and provide an easily way to address training status and monitoring training effects, and to predict performance [38]. Modeling work capacity from time to exhaustion assumes that energy releasing systems are divided only into two components (aerobic and anaerobic) with different capacity and rate of energy supply, but able to be integrated to movement energy requirements from a unique mechanical variable [39].

According to Gaesser et al. [40], Bull et al. [41], and Morton [39], the 2-parameter model has been obtained from power output $(\mathrm{P})$ and time to exhaustion (t) linear curve fitting:

$$
P \times t=A W C+C P \times t
$$

where intercept refers to AWC (anaerobic work capacity, quilojoules - $\mathrm{kJ}$ ), and the slope is $\mathrm{CP}$ (critical power, watts W). According to Bull et al. [41], a second mathematical linear model (Linear-P) could be derived by solving equation 13 for P:

$$
P=A W C \cdot(1 / t)+C P
$$

From this linear fitting for variables $\mathrm{P}$ and $\mathrm{t}$, a nonlinear 2-parameter can be described by solving it for $\mathrm{t}$ [41]:

$$
\begin{aligned}
& P-C P=A W C \cdot(1 / t) \Rightarrow \\
& {[P-C P] t=A W C \Rightarrow t=\frac{A W C}{P-C P}}
\end{aligned}
$$

which assumptions assume that $\mathrm{t} \rightarrow \infty$ when $\mathrm{P} \rightarrow 0$ and AWC is never required, or even $\mathrm{P} \rightarrow \infty$ if $\mathrm{t} \rightarrow 0$, and exhaustion takes place when AWC $=0[39,41]$. The intercept AWC represents a finite amount of work that can be performed above CP [40], i.e. the energetic reserve that is entirely depleted at the point of fatigue [39]. CP is the threshold intensity above which $\mathrm{VO}_{2 \max }$ can be elicited before exhaustion [38] (further details on CP are given later on this chapter). Then the physiological meaning of AWC is related to the factors comprising $\mathrm{O}_{2}$ deficit in muscle, and because of this it has been shown to be useful in the assessment of anaerobic capacity [40].
The usefulness of the P- $\mathrm{t}_{\text {Lim }}$ model to swimming has been impaired by the low degree of consistency in the studies concerning the measurements of the active drag [5]. Moreover, swimming doesn't match the assumption of a constant cost of transportation, since there is a change in mechanical efficiency with increasing swimming velocity due to the hydrodynamics modifications [42]. Instead, the distance-time model $\left(\mathrm{d}-\mathrm{t}_{\mathrm{Lim}}\right)$ was applied in a flume showing a strong coefficient $\left(r^{2}=0,998\right)$ to the linear adjustment between a range of swim velocity and time to exhaustion [43]. Thus:

$$
d_{\text {Lim }}=a+b \times t_{\text {Lim }}
$$

is the linear $d$ - $t_{\text {Lim }}$ plotting analogue to that implicit in Equation 13, and then could be rearranged for time, being analogue to Equation 14:

$$
v=\left(\frac{a}{t_{\text {Lim }}}\right)+b
$$

where the y-intercept in is "a", or ADC (anaerobic distance capacity, meters $-\mathrm{m}$ ), and "b" is the slope, or CV (critical velocity, $\mathrm{m} / \mathrm{s}$ ), matching the mathematical assumption that $\mathrm{t}$ $\rightarrow \infty, \mathrm{a} / \mathrm{tLim} \rightarrow 0$ and $\mathrm{v} \rightarrow \mathrm{b}$ [41].

$\mathrm{CV}$ can be defined as the intensity that can be maintained indefinitely, however there is a misinterpretation belief that CV could be sustained for a very long period of time [38]. Dekerle [38] had summarized some important aspects concerning CV:

a) it has been demonstrated to be a good indicator of the capacity of the aerobic energy system, once shown to be close to 30-min swimming-test, and highly correlated to the MLSS (maximal lactate steady state), the average 400-m swim velocity, and OBLA (onset of blood lactate accumulation);

b) its values are higher than MLSS and OBLA, and lower than the end velocity attained in an incremental test, often identified as maximal aerobic velocity;

c) time to exhaustion at $\mathrm{CV}$ is expected to be 30-40-min, which surrounds that observed at MLSS;

d) CV could be considered the upper limit of heavy intensity domain (the highest intensity that does not elicit $\mathrm{VO}_{2 \max }$ during a constant load exercise), thus inducing to central and peripheral cardio-circulatory and respiratory adaptations to exercise performed around it.

The use of power parameter (i.e., Po - overall mechanical power output) will improve meaningful of these relationships, because it's a reliable variable of muscle function in swimming. Toussaint et al. [42] related the mechanical correspondent of physiological power to a range of velocity in the pool and flume by means of hyperbolic 2-parameters equation, and reported values of $114.4 \mathrm{~W}$ and $114.5 \mathrm{~W}$, respectively. These reference values for PC in swimming are greater than the values report by Pessôa Filho et al. [44] (Table 1). This difference may be occurred once Toussaint et al. [42] have considered a high value of propelling efficiency (0.6) in the conversion of physiological power to mechanical useful equivalent. The values on Table 1 were obtained by Pessôa Filho et al. [44] from tethered swim. These authors undertook twenty four swimmers $(16.5 \pm 2.7$ years and 67.7 
$\pm 13.5 \mathrm{~kg}$ ) to active drag force (Fr) estimation, CV test calculated from distance between $200-1500 \mathrm{~m}$ and four constant bouts leading to exhaustion between 3-20min in tethered swim. The workload during bouts were fractions respective to Frp (Equation 12), and just values of tethered useful power $\left(\mathrm{Pu}_{\mathrm{Teth}}\right)$ were considered. Power delivered from tethered-crawl $\left(\mathrm{P}_{\text {TethCrit }}\right)$, free swimming velocity and distances were fitted to the time to exhaustion by linear and hyperbolic functions.

Reported values of free-crawl hydrodynamics (Table 2), from Pessôa Filho et al. [44], are in agreement with $\mathrm{Fr}=$ $25.21 \mathrm{v}^{2}$ suggested by Toussaint et al. [33]. Even, the maximal useful power in crawl-tethered $\left(\mathrm{Pu}_{\text {TetehMax }}\right)$ showed on Table $\mathbf{2}$ is only slightly higher than the predicting values for overall external mechanical output of $P_{\text {ext }}=\left\{\left[\left(21,33 \mathrm{v}^{2,34}\right) \mathrm{v}\right] /\right.$ ep\} reported by Toussaint et al. [42]. From a free front-crawl swimming velocity of $1.53 \mathrm{~m} / \mathrm{s}$, and considering ep level of $60 \%$, the values of $\mathrm{P}_{\mathrm{ext}}=156.7 \mathrm{~W}$ are given.

Table 3 and Fig. (1) reveal the greater coefficient of similarity between the index $\mathrm{Pu}_{\mathrm{Teth} C r i t}$ and $\mathrm{CV}$, recognizing the power tethered-time limit $\left(\mathrm{Pu}_{\mathrm{Teth}}-\mathrm{t}_{\mathrm{Lim}}\right)$ a suitable method to reproduce $\mathrm{P}-\mathrm{t}_{\mathrm{Lim}}$ model in swimming and thus accessing with a more reasonable accuracy of the physiological potential of the athlete.

Aerobic physiological adaptations have a potential challenge to allow swimming at faster race pace in competitive distance ranging from 200 to $1500-\mathrm{m}$, and CV was identified as good indicator of this capacity, because it has demonstrated high correlation values with these velocities [45]. Whatever, Table 4 evidences the same trend to the slope parameter of the tethered power-time limit model $\left(\mathrm{P}_{\text {Teth }}-\mathrm{t}_{\mathrm{Lim}}\right)$.

Table 1. Values from $P u_{\text {Teth }}-t_{\text {Lim }}$ Model

\begin{tabular}{|c|c|c|c|c|}
\hline & & General & Females & Male \\
\hline \hline \multirow{3}{*}{ Linear* } & $\mathrm{AWC}(\mathrm{kJ})$ & $12.90 \pm 6.61$ & $8.33 \pm 2.40$ & $14.10 \pm 6.87$ \\
\cline { 2 - 5 } & $\mathrm{Pu}_{\text {TethCrit }}(\mathrm{W})$ & $81.90 \pm 26.29$ & $45.17 \pm 4.40$ & $91.57 \pm 20.14$ \\
\cline { 2 - 5 } & $\mathrm{r}^{2}$ & $0.93 \pm 0.06$ & $0.93 \pm 0.04$ & $12.78 \pm 4.38$ \\
\hline \multirow{3}{*}{ Non-linear** } & $\mathrm{AWC}(\mathrm{kJ})$ & $19.47 \pm 8.18$ & $38.40 \pm 4.67$ & $79.70 \pm 18.32$ \\
\cline { 2 - 5 } & $\mathrm{Pu}_{\text {TethCrit }}(\mathrm{W})$ & $71.10 \pm 23.66$ & $0.95 \pm 0.04$ & $0.96 \pm 0.03$ \\
\cline { 2 - 5 } & $\mathrm{r}^{2}$ & $0.96 \pm 0.03$ & & $21.23 \pm 8.09$ \\
\hline
\end{tabular}

*Linear fitting adjust followed P-1/t $\mathrm{L}_{\text {Lim }}$ model $\left(\mathrm{P}=\mathrm{AWCxt}{ }^{-1}+\mathrm{CP}\right) .{ }^{*}$ Non-linear adjust followed hyperbolic 2-parameter $(\mathrm{t}=(\mathrm{AWC} /(\mathrm{P}-\mathrm{CP}))$.

Table 2. Parameters of Performance and Crawl Hydrodynamics

\begin{tabular}{|c|c|c|c|}
\hline Parameters & General & Female & Male \\
\hline \hline $\mathrm{v}_{\max }(\mathrm{m} / \mathrm{s})$ & $1.59 \pm 0.16$ & $1.34 \pm 0.05$ & $1.65 \pm 0.11$ \\
\hline $\mathrm{A}(\mathrm{adimensional)}$ & $25.68 \pm 4.71$ & $21.99 \pm 3.83$ & $26.65 \pm 4.51$ \\
\hline $\mathrm{Fr}_{\max }(\mathrm{N})$ & $66.72 \pm 22.13$ & $39.43 \pm 7.50$ & $73.90 \pm 18.76$ \\
\hline $\mathrm{Pu}_{\text {TethMax }}(\mathrm{W})$ & $135.73 \pm 44.93$ & $74.61 \pm 10.48$ & $151.82 \pm 35.28$ \\
\hline
\end{tabular}

Ps: $\mathrm{V}_{\max }$ is the maximal swim front-crawl swimming velocity for $13-15 \mathrm{~m}$; A is the proportionality coefficient; $\mathrm{Fr}_{\max }$ is the active drag force measured according to Toussaint $e$ al (1998) at $\mathrm{v}_{\max }$; and $\mathrm{Pu}_{\mathrm{TethMax}}$ is the maximal useful power corresponding to $\mathrm{Fr}_{\max }$ loading in tethered front-crawl swimming. Reprinted with permission.

Table 3. Correlation (Pearson Coefficient) Between Values of Power-Time Limit Parameters and Those of Velocity-Time Limit

\begin{tabular}{|c|c|c|c|c|c|}
\hline & & CV (Linear) & ADC (Linear) & CV (Non-Linear) & ADC (Non-Linear) \\
\hline \multirow{3}{*}{ P-t $t_{\text {Lim }}$ model } & $\mathrm{Pu}_{\text {TethCrit }}$ (linear) & $0.812^{* *}$ & -0.393 & $0.776^{* *}$ & 0.013 \\
\hline & AWC (linear) & $0.437 *$ & -0.036 & 0.425 & 0.137 \\
\hline & AWC (non-linear) & $0.448^{*}$ & -0.021 & $0.487 *$ & 0.013 \\
\hline
\end{tabular}

$* \mathrm{p} \leq 0.05 . * * \mathrm{p} \leq 0.01$. 

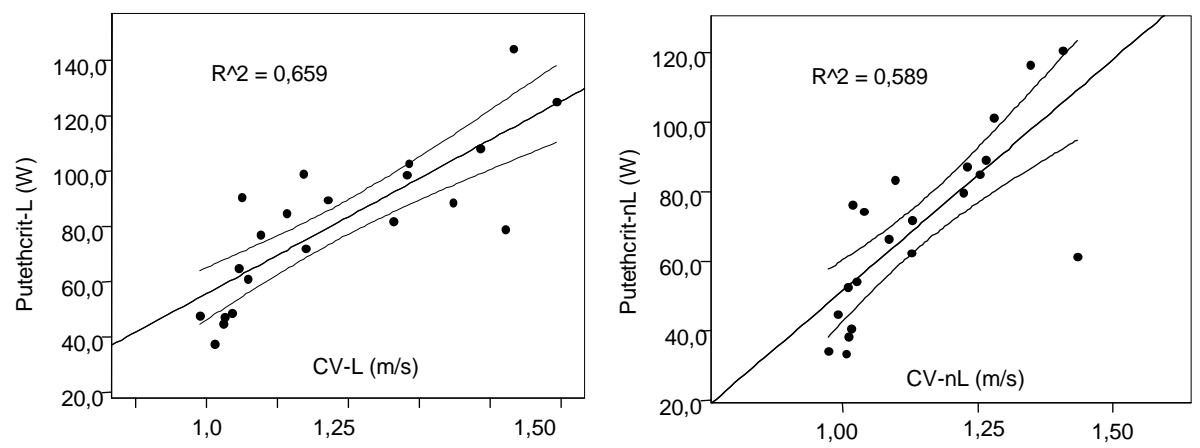

Fig. (1). Dispersion around linear fitting $\mathrm{Pu}_{\text {TethCrit }}$ to $\mathrm{CV}$ (linear and non-linear).

Table 4. Correlations (Pearson Coefficients) Between the Values of $\mathrm{Pu}$ TethCrit (Linear and Non-Linear) and the Competitive Performances in Free Swimming Condition

\begin{tabular}{|c|c|c|}
\hline Distance Performed & $\mathbf{P u}_{\text {TethCrit }}($ Linear $)$ & $\mathbf{P u}_{\text {TethCrit }}($ Non-Linear $)$ \\
\hline \hline $200-\mathrm{m}$ & $-0.785^{*}$ & $-0.806^{*}$ \\
\hline $400-\mathrm{m}$ & $-0.752^{*}$ & $-0.754^{*}$ \\
\hline $800-\mathrm{m}$ & $-0.810^{*}$ & $-0.822^{*}$ \\
\hline $1500-\mathrm{m}$ & $-0.831^{*}$ & $-0.852^{*}$ \\
\hline
\end{tabular}

$\mathrm{p} \leq 0.01$.

\section{CONCLUSION}

The use of active drag force as a load parameter in tethered swimming increased its specificity with the freeswimming condition, and the power variable measured in this tethered condition is quite representative of mechanical power produced during free-swimming. Thus, the powertime limit model could be reproducible in swimming, but this reliability concerning the similarity of the $\mathrm{Pu}_{\text {TethCrit }}$ with the other indexes of aerobic capacity, training intensity and control of training effects is an open field to be explored in further studies.

\section{REFERENCES}

[1] Clarys JP. In: Terauds J, Bedinfield Ew, Ed. Human morphology and hydrodynamics. Swimming III 1979; 8: 3-41.

[2] Lewillie L. In: Hollander AP Huijing PA deGroot G, Eds. Research in swimming: historical and scientific aspects. Biomech Med Swim 1983; 14: 6-16.

[3] Nigg BM. In: Hollander AP Huijing PA deGroot G, Eds. Selected methodology in biomechanics with respect to swimming. Biomech Med Swim 1983; 14: 73-80.

[4] Martin RB, Yeater RA, White MK. A simple analytical model for the crawl stroke. J Biomech 1981; 14(8): 539-48.

[5] Hay JG. In: Ungerechts BE, Wilke K, Reischle K, Eds. The status of research on the biomechanics of swimming. pp. 03-14. Swimming Science V 1988; 18: 3-14.

[6] Adrian, MJ. In: Hollander AP Huijing PA deGroot G, Eds. Biomechanics and mathematical modeling. Biomech Med Swim 1983; 14: $142-153$.

[7] Yeater RA, Martin RB, White MK, et al. Tethered swimming forces in the crawl, breast and back strokes and their relationship to competitive performance. J Biomech 1981; 14(8): 527-37.

[8] Dopsaj M, Matkovic I, Zdravkovic I. The relationship between $50 \mathrm{~m}$ - freestyle results and characteristics of tethered forces in male sprint swimmers: a new approach to tethered swimming test. Phys Education Sport 2000; 1(7): 15-22.
[9] Huijing PA, Hollander AP, deGroot G. In: Hollander AP Huijing PA deGroot G, Eds. Efficiency and specificity of training in swimming: an Editorial. Biomech Med Swim 1983; 14: 1-6.

[10] Cabri JMH, Annemans L, Clarys JP, et al. In: Ungerechts BE, Wilke K, Reischle K, Eds. The relation of stroke frequency, force, and emg in font crawl tethered swimming. Swimming Science V 1988: 18:183-189.

[11] Holmér I. In: Hollander AP, Huijing PA, deGroot G, Eds. Energetics and mechanical work in swimming. Biomech Med Swim 1983; 14: 154-164.

[12] Bollens E, Annemans L, Vaes W, et al. In: Ungerechts BE, Wilke K, Reischle K, Eds. Periferical EMG comparison between fully tethered and free front crawl swimming. pp. 173-182. Swimming Science V 1988; 18.

[13] Costill DL, King DS, Holdren A, et al. Sprint speed vs swimming power. Swim Tech 1983; 20-22.

[14] Kjendlie P-L, Thorsvald K. In: Vilas-Boas JP, Alves F, Marques A, Eds. A tethered swimming power test is high reliable. Biomechanics and Medicine in Swimming X. Rev Port Cienc Desp 2006; 6 (2): 231-235.

[15] Vorontsov A, Popov O, Binevsky D, et al. In: Vilas-Boas JP, Alves F, Marques A, Eds. The assessment of specifcis strength in welltrained male athletes during tethered swimming in the swimming flume. Biomechanics and Medicine in Swimming X. Rev Port Cienc Desp 2006; 6(2): 275-277.

[16] Rouard AH, Aujouannet YA, Hintz F, et al. In: Vilas-Boas JP, Alves F, Marques A, Eds. Isometric force, tethered force and power ratios as tools for the evaluation of technical ability in freestyle swimming. Biomechanics and Medicine in Swimming X. Rev Port Cienc Desp 2006; 6(2): 249-250.

[17] Petriaev A, Kleshnev I. In: Vilas-Boas JP, Alves F, Marques A, Eds. Diagnostic, training and realization of strength condition of swimmer with use of feedback diagnostic simulator art. Biomechanics and Medicine in Swimming X. Rev Port Cienc Desp 2006; 6(2): 244-246.

[18] Maglischo EW, Maglischo CW, Zier DJ, et al. The effect of sprintassisted and sprint-resisted swimming on stroke mechanics. J Swim Res 1985; 1(2): 27-33.

[19] Chatard JC, Collomp C, Maglischo EW, et al. Swimming skill and stroking characteristics of front crawl swimmers. Int J Sports Med 1990; 11: 156-161.

[20] Millet GP, Chollet D, Chalies S, et al. Coordination in front crawl in elite triathletes and elite swimmers. Int J Sports Med 2002; 23: 99-104.

[21] Pessôa Filho DM, Monteiro HL. Efeitos da força muscular sobre a mecânica de nado e sobre a velocidade do crawl após treinamentos com peso. (Muscle force influence upon swimming mechanical parameters and crawl velocity after specific weight training). In: XI Congresso Brasileiro de Biomecânica, 2005, João Pessoa. Anais do XI Congresso Brasileiro de Biomecânica, 2005.

[22] Toussaint HM, Ross PE, Kolmogorov S. The determination of drag in front crawl swimming. J Biomech 2004; 37: 1655-1663.

[23] Tousssaint HM, Vervoorn K. Effects of specific high resistance training in the water on competitive swimmers. Int J Sports Med 1990; 11: 228-233.

[24] Toussaint HM, Carol a, Kranenborg H, et al. Effect of fatigue on stroking characteristics in an arms-only 100-m front-crawl race. Med Sci Sports Exerc 2006; 38(9): 1635-1642. 
[25] Wakayoshi k, D’Aquisto LJ, Cappaert JM, et al. Relationship between oxygen uptake, stroke rate and swimming velocity in competitive swimming. Int J Sports Med 1995; 16(1): 19-23.

[26] deGroot G, Ingen Schenau GV. In: Ungerechts BE, Wilke K, Reischle K, Eds. Fundamentals mechanics applied to swimming: technique and propelling efficiency. Swimming Science V 1988; 18: 17-30.

[27] Toussaint HM, van der Helm FCT, Elzerman JR, et al. In: Hollander AP, Huijing PA, DeGroot T G, Eds. Power Balance Applied to Swimming. Biomech Med Swim 1983; 14: 258-263.

[28] Schleihauf RE. In: Terauds J, Bedingfield EW, Eds. A hydrodynamic analysis of swimming propulsion. Swimming III 1979; 3.

[29] Berger MAM, Hollander AP, deGroot G. Technique and energy losses in front crawl swimming. Med Sci Sports Exerc 1997; 29(11): 1491-1498.

[30] Miller DI. Biomechanics of Swimming. In: Wilmore JH, Koegh JF, Eds. Exerc Sports Sci Rev 1975; 03.

[31] Barthels KM. Swimming biomechanics: resistance and propulsion. Swim Tech 1977; 14: 66-72.

[32] Barret DS, Triantafyllou MS, Yue DKP, et al. Drag reduction in fish-like locomotion. J Fluid Mech 1999; 392: 183-212.

[33] Toussaint HM. Differences in Propelling Efficiency Between Competitive and Triathlon Swimmers. Med Sci Sports Exerc 1990; 22(03): 409-415.

[34] Schleihauf RE. In: Hollander AP, Huijing PA, DeGroot G, Eds. Specificity of Strength Training in Swimming: a Biomechanical ViewpointBiomech Med Swim. Human Kinetics, Champaing, IL 1983: 184-191.

[35] Olbrecht J, Clarys JP. In: Hollander AP, Huijing PA, DeGroot G, Eds. EMG of specific dry land training for the front crawl. Biomech Med Swim Champaign: Human Kinetics 1983; 14: 136141 .
[36] Magel JR. Propelling force measured during tethered swimming in the four competitive swimming styles. Res Quarterly 1970; 41: 6874.

[37] Craig AB, Boomer WF. Relationship between tethered and free swimming the front crawl stroke. J Biomech 1980; 13.

[38] Dekerle J. In: Vilas-Boas JP, Alves F, Marques A, Eds. The use of critical velocity in swimming: a place for critical stroke rate? Biomechanics and Medicine in Swimming X. Rev Port Cienc Desp 2006; 6(2): 201-205.

[39] Morton RH. The critical power and related whole-body bioenergetic models. Eur J Appl Physiol 2006; 96: 339-354.

[40] Gaesser GA, Carnevale TJ, Garfinkel A, et al. Estimation of critical power with nonlinear and linear models Med Sci Sports Exerc 1995; 27: 1430-1438.

[41] Bull AJ, Housh TJ, Johnson GO, et al. Effect of mathematical modeling on the estimation of critical power. Med Sci Sports Exerc 2000; 32: 526-530.

[42] Toussaint HM, Wakayoshi K, Hollander AP, et al. Simulated front crawl swimming performance related to critical speed and critical power. Med Sci Sports Exerc 1998; 30(1): 144-151.

[43] Wakayoshi K, Ikuta K, Yoshida T, et al. Determination and validity of critical velocity as an index of swimming performance in the competitive swimmer. Eur J Appl Physiol Occup Physiol 1992; 64: 153-157.

[44] Pessôa Filho DM, Nascimento EP, Denadai BS. Validação do modelo potência-tempo limite no $\mathrm{crawl}$-atado pelas relações com os modelos distância/tempo limite e velocidade/tempo limite (Validity of the power-time to exhaustion model in the tethered-crawl swim from relationships to the distance and velocity-time to exhaustion models). Rev Bras Cienc Esporte 2008; 29(2): 95-114.

[45] Hill DW, Ferguson CS. A physiological description of critical velocity. Eur J Appl Physiol 1999; 79: 290-293.

Received: March 27, 2008

Revised: May 20, 2008

Accepted: May 28, 2008

(c) Pessôa Filho and Denadai; Licensee Bentham Open.

This is an open access article distributed under the terms of the Creative Commons Attribution License (http://creativecommons.org/licenses/by/2.5/), which permits unrestrictive use, distribution, and reproduction in any medium, provided the original work is properly cited. 